\section{SIMLEN: A response sampling algorithm to simulate some features of learning}

\author{
S. A. ROBERTSON \\ Manchester Polytechnic, Manchester, England M15 5FJ
}

A BASIC program is presented which can simulate some features of learning, including the partial reinforcement effect. The operation of the algorithm depends upon the use of a response sampling procedure.

As Apter (1970) points out, it is possible to simulate learning under the law of effect using a computer without great difficulty. In fact, the basic requirement for such a simulation is a response generator which produces responses on the basis of differential probabilities, all of which are linked to the magnitude of reinforcement applied previously to the responses themselves. Thus, a response $\mathrm{Ri}$ has a likelihood $\mathrm{pRi}$ dependent upon the extent to which it has been reinforced in the past.

What proves more difficult is the attempt to provide a simulation which has greater breadth in being able also to manifest other well known learning phenomena.

SIMLEN is a program designed by the author as a simple learning simulator capable of showing susceptibility not only to positive or negative reinforcement and extinction, but also to the partial reinforcement effect (PRE). SIMLEN may be operated in any of three modes depending upon whether an extinction, partial reinforcement, or continuous reinforcement run is required. In each mode, the parameters of the run may be input by the user.

The algorithm underlying SIMLEN involves a response sampling procedure based upon a store of response elements. Consider, for example, a repertoire of say 10 responses, R0 to R9. The basic notion is one of a store in which are contained a number of response elements representing each of the 10 responses. At the outset, equal numbers of elements represent the 10 different responses. The generation of a response is operationalized as the random selection and replacement of an element from store. If an element representing R3 is selected, R3 is assumed to be the "behavior" generated by the model. If the selection of an element is random with respect to the total store content $\mathrm{T}$, then each element will have a probability of $1 / \mathrm{T}$. Consequently, if there are $\mathrm{Ni}$ elements associated with response $\mathrm{Ri}$, the probability of generation of $\mathrm{Ri}$ will be equal to $\mathrm{Ni} / \mathrm{T}$. If we suppose at the outset that five elements representing each of the 10 responses make up the total store, then $\mathrm{T}=50$ and for any response $\mathrm{Ri}, \mathrm{pRi}=5 / 50$, or .1 .

In continuous reinforcement mode (CR), positive and negative reinforcement can be directly operationalized as the addition or subtraction of elements to or from store when the "target response" has been generated. In partial reinforcement mode (PR), the proportion of occasions on which this occurs will be less than $100 \%$, while in extinction mode (EX) neither of these processes need be operationalized at all.

A random component has been added to the model so as to allow for a more realistic representation of individual differences. In fact, however, it is the addition of this random component that allows the simulation of extinction and PRE. On those occasions when the target response is not generated, a small "noise" addition (usually one element as determined by the user) is made to the store. The response which this represents is chosen randomly from among the 10 on an equal likelihood basis.

In CR and PR mode the simulation of the effects of positive and negative reinforcement is relatively direct. Adding to or subtracting from the response store produces slow increases or decreases in the target response probability, subject to the size of the random "noise" addition. In EX mode the random component of the model acts in isolation to slowly reduce the importance of differences between the numbers of elements representing each response in the repertoire.

As might be anticipated, a larger number of trials is required normally to reach any given criterion target response probability under $P R$ mode than under CR mode. In the event, the consequence of this is that when reached, the criterion probability is numerically identical although fractionally different in the two modes PR and CR. Hence, having reached a criterion probability of .6 , this might be based on 54 target response elements in a total store of 90 in PR mode, while being based on 42 target elements in 70 in CR mode. It is this fractional difference (between 54/90 and 42/70) which produces a situation where extinction to any specified level takes longer in the former (PR) case. If we were to begin an extinction run in both cases, the addition of one nontarget element to store would cause in the first situation (PR) a drop in target response probability of .0066 (i.e., to 54/91). In the CR case the drop would be .0085 (i.e., to $42 / 71$ ). We would, therefore, expect to find a steeper extinction curve in the latter case (CR) and, as a result, PRE.

Program Input. The first input (Line 30) to SIMLEN is the mode of operation for the run (EX, CR, or PR). The next four inputs (Line 40) include first the number of trials required and second, the criterion target response probability at which to stop the run. Either factor can cause run termination. Third, the size of the noise addition (usually 1 ) is input and fourth, the target response number (between 0 and 9 inclusive). If EX mode has been selected, the number of response elements associated with each of the 10 responses is then input in a stepwise manner starting with R0. If CR or PR mode has been selected, the next input (Line 60) is of the size of the reinforcement addition 
(usually 2 or 3 ). If PR mode has been selected, a final input is necessary (Line 80), namely, the percentage reinforcement required. In PR mode this final parameter can be understood as the probability that reinforcement will be delivered after the target response has been generated.

Program Output. SIMLEN outputs a trial-by-trial monitor of the numbers of response elements associated with each response during the course of a run together with a final breakdown of the run characteristics.
This breakdown includes the number of trials reached, the key or target response, the final response probability, and the number of reinforcements applied.

Language and Computer. SIMLEN was written in BASIC and developed using an ICL 1902S computer. Availability. A listing of SIMLEN together with sample output is available at no cost upon request from the author at the School of Psychology, Manchester Polytechnic, Gaythorn Annexe, River Street, Manchester, England M15 5FJ.

Table 1

SIMLEN: A BASIC Leaming Simulator

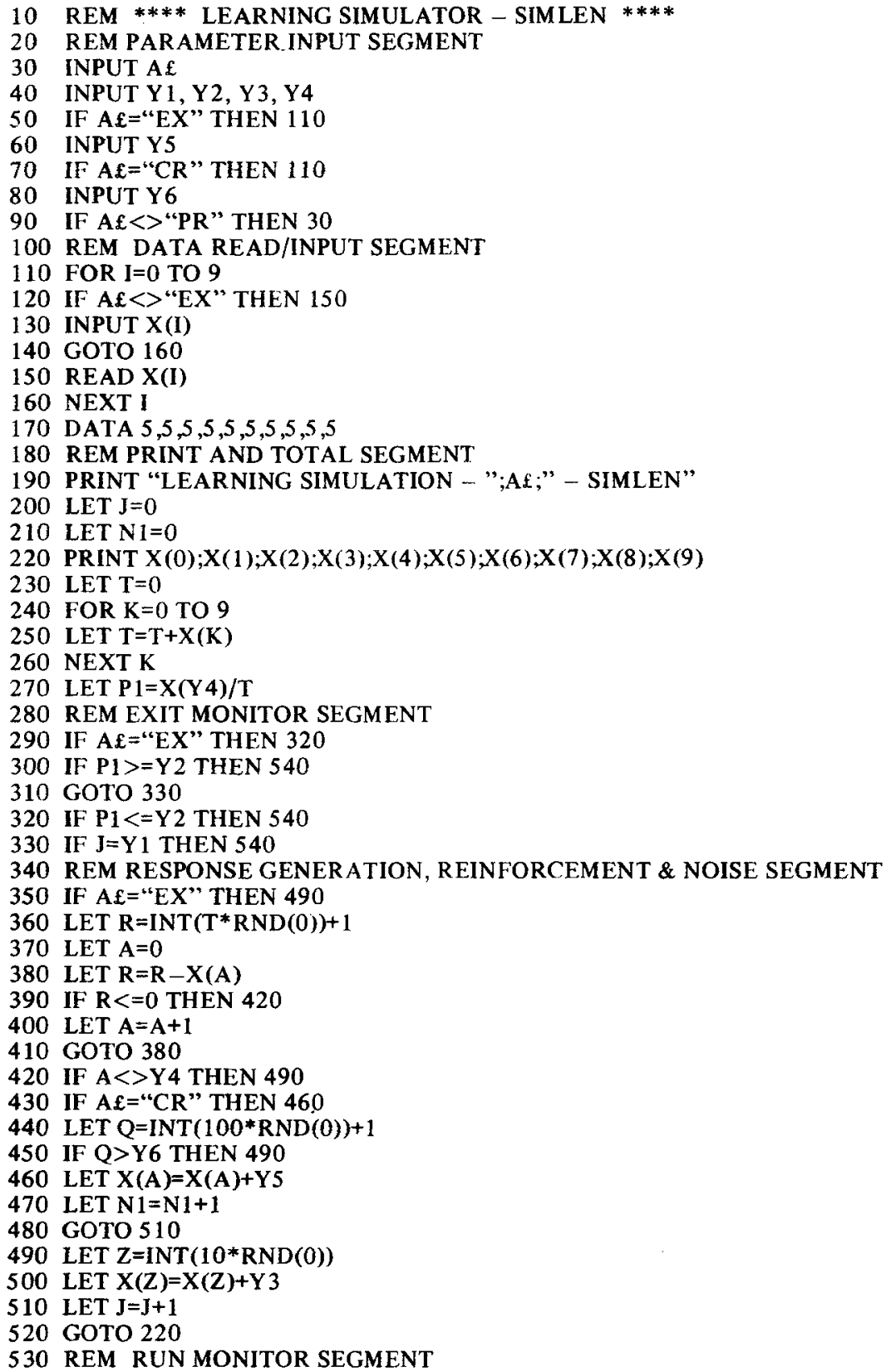


540 PRINT "RUN COMPLETE - ";A£

550 PRINT "TRIALS = ";J

560 PRINT "KEY RESPONSE = ";Y4

570 PRINT "RESPONSE PROBABILITY = ";P1

580 PRINT "NUMBER OF REINFORCEMENTS APPLIED = ";N1

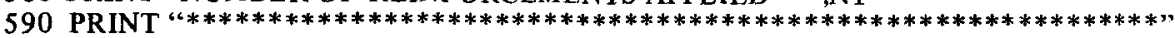

600 STOP

610 END

\section{REFERENCE}

APTER, M. J. The computer simulation of behaviour.

London: Hutchinson University Library. 1970.

(Accepted for publication July 12, 1977.) 